\title{
Generationenbeziehungen im Alter: Türkische Familien in der Türkei und in Westeuropa
}

\author{
Intergenerational relationships in old age: Turkish families in Turkey and \\ in Western Europe
}

\begin{abstract}
:
Dieser Beitrag nähert sich der Frage nach den Auswirkungen einer internationalen Migration auf die Generationenbeziehungen älterer Menschen aus einer neuen Perspektive: Verglichen werden die Beziehungen in türkeistämmigen Familien in Westeuropa mit Familien in der Türkei sowie, als dritte Gruppe, transnationalen Familien. Die Datengrundlage bildet die internationale LineUpStudie „Migration Histories of Turks in Europe“. Zielvariablen sind familienbezogene Werteinstellungen sowie Kontakthäufigkeit und gegenseitige Unterstützungsleistungen aus Sicht der erwachsenen Kinder. Migrantenfamilien zeigen in den Verhaltensmustern intensivere Beziehungen als $\mathrm{Fa}$ milien in der Türkei, allerdings stellen diese Unterschiede nahezu vollständig Kompositionseffekte dar. Unterschiede in den Werteeinstellungen, mit geringerer normativer Solidarität in Migrantenfamilien, bleiben dagegen auch in multivariaten Analysen tendenziell bestehen. Transnationale Familien weisen die geringste Generationensolidarität auf, was auf die große Wohnentfernung zurückzuführen ist. Die Befunde geben tendenziell Hinweise auf einen Wertewandel in der Migration bei weitgehender Kontinuität der Verhaltensmuster.
\end{abstract}

Schlagwörter: Migration, Türkei, Generationenbeziehungen, Migrantenfamilie, Ältere Migranten, Wertewandel

\begin{abstract}
This paper explores the consequences of international migration on family relationships of elderly migrants from a new perspective: It compares intergenerational relationships among migrants from Turkey who live in Europe with those among non-migrants who never went abroad and, as a third group, transnational families. This study draws from the international LineUp Survey "Migration Histories of Turks in Europe". Dependent variables are the frequency of contact, mutual support exchange patterns and family values as reported by the adult children. Findings indicate more intense intergenerational relationships in migrant families as compared to families in Turkey, but lower agreement with norms on intergenerational solidarity among the former. Whereas differences in behavior can be explained almost completely by compositional differences, multivariate analyses suggest persisting divergences in attitudes. Transnational families show the lowest degree of intergenerational solidarity which can be explained by the large spatial distance. By tendency, the findings indicate a change in values, but overall continuity in behavior patterns in the course of an international migration.
\end{abstract}

Key words: migration, Turkey, intergenerational relationships, migrant family, elderly migrants, value change 


\section{Einleitung}

Die Diskussion um den Wandel familiärer Strukturen im Zuge einer internationalen Migration berücksichtigt unter anderem die Generationenbeziehungen älterer Menschen. Dieses Thema hat mit den umfangreichen internationalen Migrationsprozessen und dem demographischen Alterungsprozess v.a. in den Industrieländern deutlich an Aufmerksamkeit gewonnen (Treas/Marcum 2009; Silverstein/Attias-Donfut 2010). Selten ist es bisher möglich, die theoretisch formulierten Erwartungen hinsichtlich von Migrationseffekten an geeigneten Daten zu überprüfen. Viele Studien vergleichen Familienmuster in Migrantenfamilien (unterschiedlicher Herkunft) mit denen der einheimischen Bevölkerung im Zielland, um die „Spezifika“ in der Migrationssituation herauszuarbeiten (z.B. Burr/Mutchler 1999; de Valk/Schans 2008). Nach Kontrolle sozioökonomischer und anderer Variablen werden bestehende Differenzen zwischen Migranten und Einheimischen der „Herkunftskultur“ oder der „Migrationserfahrung“ zugeschrieben. Ein Problem dieses Ansatzes besteht darin, dass eine Trennung von Herkunfts- und Migrationseffekten kaum möglich ist und über die Bedeutung der Migration nur eingeschränkte Aussagen getroffen werden können. Alternativ, aber bisher nur selten, wird zwischen Migranten und Nichtmigranten derselben Herkunft unterschieden (z.B. Nauck 1997; ähnlich: Cong/Silverstein 2011). Differenzen sind dann als Hinweis auf Migrationseffekte zu interpretieren, ohne dass allerdings Mechanismen der selektiven Migration vollständig ausgeschlossen werden können. Dabei ist zu berücksichtigen, dass Wandel in den Familien sowohl in der Migration (durch den Kontextwechsel und Akkulturationsprozesse) als auch im Herkunftsland (sozialer Wandel) stattfinden kann (Nauck 2002).

Der Beitrag untersucht die Unterschiede in den Generationenbeziehungen zwischen internationalen Migranten und Nichtmigranten derselben Herkunftsregion auf Basis der Daten des LineUp-Surveys (Baykara-Krumme/Nauck 2011). Im Mittelpunkt des Interesses stehen die in verschiedenen Ländern Westeuropas alt gewordenen ehemaligen Arbeitsmigranten aus der Türkei mit ihren Kindern, die zwischen 1960 und 1974 im Rahmen von staatlichen Anwerbeabkommen auswanderten, und die „peers“ mit ihren Familien aus denselben Herkunftsregionen, die die Türkei nicht verlassen haben. ${ }^{1}$ Als dritte Gruppe werden transnationale Familien betrachtet, in denen die Eltern im Herkunftsland und die Kinder in Westeuropa leben, weil die Kinder später eigenständig dorthin emigrierten oder die Eltern ohne die Kinder in die Türkei zurückkehrten. Im Fokus stehen Unterschiede in den Generationenbeziehungen zwischen diesen drei Gruppen und die Rolle von „Migrationseffekten“. Untersucht werden in Anlehnung an das Modell der intergenerationalen Solidarität (Bengtson/Robert 1991) die assoziative Solidarität (Kontakthäufigkeit), zwei Dimensionen der funktionalen Solidarität (finanzielle und kognitive Hilfe) und die normative Solidarität aus Sicht der Kinder.

1 Die Türkei schloss entsprechende Abkommen bzw. Verträge u.a. mit Deutschland (1961), Belgien, den Niederlanden und Österreich (jeweils 1964), Frankreich (1965) und Schweden (1967). Weitere Länder ermöglichten die Einreise von Arbeitsmigranten ohne ein formelles Abkommen (z.B. Dänemark, Schweiz). Insgesamt verließen über 800.000 Menschen die Türkei als offiziell registrierte Arbeitsmigranten, davon 81\% nach Deutschland (Akgündüz 2008: 61, 79). 
2. Theoretischer und empirischer Hintergrund

\subsection{Erklärungsansätze}

Die internationale und kulturvergleichende Forschung führt Unterschiede in „Familienkulturen", die sich in Generationenbeziehungen niederschlagen, auf historisch unterschiedliche Erb- und Heiratsregimes, ökonomische Entwicklungspfade oder -stadien, wohlfahrtsstaatliche Entwicklungen oder kulturell-religiöse Einflüsse zurück (z.B. Goode 1970; Kağıtçıbaş1 1996; Reher 1998; Hofstede 2001; Thornton 2005; Albertini et al. 2007; Blome et al. 2008). Hier geht es um die Frage, ob internationale Migranten Einstellungs- und Verhaltensmuster als kulturelles Erbe „importieren“ und beibehalten oder sich diese angesichts veränderter Rahmenbedingungen wandeln, und dann möglicherweise in „hybridized forms“ (Silverstein/Attias-Donfut 2010: 180) resultieren.

Nauck unterscheidet zwei Erklärungsansätze für die familialen Migrationsfolgen (Nauck 1988). In der makrosoziologisch-modernisierungstheoretischen Perspektive hängt ein Wandel mit dem Wechsel von einer agrarisch-segmentären Gesellschaftsform (mit kollektivistisch-interdependenten Wertorientierungen wie in der Türkei mit ihrem überwiegend patrilineal-dependenzverwandtschaftlichen Familiensystem) zu einer funktional komplexen Gesellschaftsform (mit individualistisch-independenten Wertorientierungen wie in den affinalverwandtschaftlich organisierten Ländern Westeuropas) zusammen. Nach der strukturell-funktionalen Tradition von Durkheim (1921) und Parsons (z.B. mit Bales 1956) bedingt eine durch ein allgemeines Wertsystem stabilisierte Sozialstruktur die Familienstruktur. Eine Migration stellt dann einen „mikrosozialen Beschleunigungsfaktor für die ,Modernisierung' der Familie dar (...), der die Familie zu einem akkulturativen Konformismus, shift' von den Werten der Herkunfts- zu denen der Aufnahmegesellschaft ,zwingt" “ (Nauck 1988: 506). Dem Wertewandel folgen Veränderungen in der Familie, die angesichts antagonistischer Wertvorstellungen als konflikthaft postuliert werden. Individualistisch-handlungstheoretische Ansätze sehen die Ursachen für Veränderung dagegen in den veränderten kontextuellen Bedingungen und Opportunitätsstrukturen, die bei den situational-rational handelnden Individuen zu veränderten Handlungsentscheidungen führen. Familiäre Veränderungen können mit einem akkulturativen Wertewandel einhergehen. Dieser ist aber weder notwendig noch zwangsläufig, sondern von zusätzlichen Randbedingungen abhängig (Nauck 1988).

Als Opportunitätsstruktur für finanzielle Hilfen zwischen den Generationen ist z.B. bedeutsam, in welchem Umfang wohlfahrtsstaatliche Institutionen Risikolagen abdecken (Albertini et al. 2007). Dabei müssen umfangreiche wohlfahrtsstaatliche Strukturen die familiale Solidarität keineswegs verdrängen (Künemund/Vogel 2006). Während die Türkei dem Typus des familialistischen Wohlfahrtsregimes zuzuordnen ist, das eine starke familiale Solidaritätsstruktur erfordert, zählen die westeuropäischen Zielländer der türkischen Arbeitsmigration zu den ausgebauten Wohlfahrtsregimen, in denen andere Formen familialer Solidarität, wie z.B. finanzielle Transfers an erwachsene Kinder, möglich werden. Weitere Rahmenbedingungen, z.B. für kognitive Hilfe zwischen den Generationen, ergeben sich aus der (Nicht-)Existenz alternativer sozialer (Verwandtschafts-)Netzwerke in der Migration. Schließlich wirken sich neue Möglichkeiten (oder Grenzen) der Bildungs- und Arbeitsmarktpartizipation in der Migration in Form veränderter Bedürfnis- 
und Ressourcenstrukturen auf die intergenerationalen Beziehungen aus (vgl. Milewski in diesem Band).

Assimilationskonzepte postulieren im Kontext der „race-relation-cycles“ intergenerational unterschiedlich intensiv verlaufende Akkulturationsprozesse (vgl. Park 1964), mit entsprechenden Entfremdungspotenzialen zwischen den Familienmitgliedern (Schrader et al. 1976). Diese einseitige Sicht, wonach sich in der Migration die „normalen“ intergenerationalen Differenzen verstärken, gar in ausgeprägten Generationenkonflikten resultieren, wurde inzwischen vielfach differenziert. Vor dem Hintergrund der Theorie der "segmented assimilation“ formulieren Autoren wie Zhou (1997) oder Portes/Rumbaut (2001) als Alternative zur „generationalen Dissonanz“ die Option der „generationalen Konsonanz“ oder „konsonanten Akkulturation“, bei der eingewanderte Eltern und ihre Kinder sich im Akkulturationstempo und -niveau durchaus ähneln. Eine (kulturelle) Entfremdung zwischen den Generationen tritt möglicherweise erst zwischen den Einwanderern und ihren Enkeln ein (Silverstein/Chen 1999) oder bei transnational lebenden Familien (Portes 2003).

Außerdem wurde darauf verwiesen, dass sich die familiären Bindungen in der Migrations- und Minoritätensituation auch intensivieren können. In Folge von Diskriminierungserfahrungen kann die Familie wichtige Kompensationsfunktionen übernehmen (z.B. Herwartz-Emden 2000). Transmissionsanstrengungen der Eltern werden möglicherweise bewusst intensiviert, wenn ethnisch-kulturelle Institutionen fehlen oder eine (mehr oder minder konkrete) Rückkehrabsicht besteht (Phalet/Schönpflug 2001; Nauck 2002). Dabei verändern sich die Richtung der Transmission und die Formen der Hilfe über den Lebensverlauf (Bengtson/Allen 1993). Während generell in der Migration im Vergleich zum Herkunftsland aufgrund des fremden Umfelds und der erforderlichen Sprachkenntnisse von einem größeren Hilfebedarf auszugehen ist, kann sich diese Bedürftigkeit im Alter verstärken. Die für die Älteren postulierte Rückorientierung auf die Herkunftskultur resultiert auch aus den abnehmenden Opportunitäten für interkulturelle Kontakte und entsprechende Fähigkeiten (wie Sprachkompetenzen). Ein Wiederaufleben der Ethnizität im Alter kann höhere Bedarfe und entsprechende Erwartungen an die Kinder implizieren (Dietzel-Papakyriakou 1993). Allerdings ist von einer solchen Konvergenz der Muster im Alter angesichts der großen interindividuellen Differenzen über den Lebenslauf keineswegs auszugehen (Matthäi 2005), und Ältere ihrerseits können nicht zuletzt aufgrund ihrer Lebenserfahrungen in der Migration eine wichtige (kognitive) Unterstützungsressource für ihre Kinder sein.

Die genannten Mechanismen postulieren einerseits Wandel in der Migration, der als Prozess der familialen Solidarisierung oder De-Solidarisierung konzeptionalisiert werden kann (Baykara-Krumme et al. 2011), beinhalten aber auch Momente der Konstanz und „cultural retention“ (Dietzel-Papakyriakou 1993). Möglicherweise sind Veränderungen im Herkunftskontext insgesamt größer, ist dieser doch (ebenfalls) von Veränderung geprägt. Kennzeichnend im Fall der Türkei in den letzten Dekaden sind Modernisierungsprozesse mit Industrialisierung, Urbanisierung und Binnenmigration, demographischem Wandel und wachsender Bildungspartizipation breiter Bevölkerungsschichten (Nauck/ Klaus 2005). Dabei muss von einem regional unterschiedlich stark ausgeprägten Wandel ausgegangen werden (El-Menouar/Fritz 2009), der weniger linear verläuft als modernisierungstheoretisch angenommen. Kağıtçıbaşı postuliert in dem von ihr vorgeschlagenen „family model of emotional interdependence“ Veränderung mit gleichbleibend hoher oder 
sogar steigender emotionaler Interdependenz bei abnehmender Bedeutung der materiellen Abhängigkeit in der Familie (z.B. Kağıtçıbaşı 1996; Ataca et al. 2005).

\subsection{Forschungsstand}

Intergenerationale Solidarität in der Lebensphase Alter wird in der Forschung zur Türkei als Teilaspekt intensiver inter- und intragenerationaler Austauschprozesse über den gesamten Lebenslauf beschrieben, die den Transfer von Normen, Werten und Traditionen (von den Älteren an die Jüngeren) ebenso einschließen wie die instrumentelle, finanzielle und emotionale Hilfe an bedürftige Familienmitglieder (Kalaycıŏlu/Rittersberger-T1lıç 2000). International vergleichende quantitative Studien zeigen eine häufigere Koresidenz Älterer mit ihren verheirateten Söhnen in der Türkei als z.B. in Deutschland, ebenso verhält es sich mit den Unterstützungserwartungen der Mütter an ihre erwachsenen Töchter. Gespräche mit erwachsenen Töchtern bzw. mit Müttern über persönliche Dinge oder Diskussionen über Probleme finden dagegen in der Türkei und in Deutschland ähnlich häufig statt (Nauck/Suckow 2006). Aytaç findet mit zunehmender sozioökonomischer Entwicklung innerhalb der verschiedenen Regionen in der Türkei zwar einen Rückgang der intergenerationalen Koresidenzraten, aber weiterhin große Nähe durch räumlich nahes $\mathrm{Zu}$ sammenleben (1998: 251). Schon frühere Studien zeigten die große emotionale Bindung und enge Kontaktdichte in den Beziehungen zu Älteren über die Grenzen der Haushalte hinweg, die faktisch die Funktion von „extended families“ aufweisen (Duben 1982).

Die starke Familienorientierung zeigt sich auch bei türkeistämmigen Migranten in Westeuropa: Im Vergleich mit der einheimischen Bevölkerung, z.B. in den Niederlanden und Deutschland, sind sie deutlich stärker familiär orientiert (Merz et al. 2009; de Valk/ Schans 2008) und haben häufiger Kontakt mit ihren Eltern (Schans/Komter 2010; Baykara-Krumme 2008; Hubert et al. 2009). Kognitive bzw. emotionale Unterstützung leisten Türkeistämmige dagegen seltener an ihre Eltern (Baykara-Krumme 2012; Schans/Komter 2010), obwohl erwachsene Kinder als potenzielle kognitive Unterstützungsressourcen ähnlich wichtig sind. Deutlich häufiger als bei Einheimischen werden Kinder als Gesprächspartner genannt, wenn man auch jene, die mit im Haushalt leben, einbezieht. Denn intergenerationale Koresidenz mit erwachsenen Kindern ist bei Türkeistämmigen signifikant häufiger, und bleibt als Besonderheit z.B. gegenüber Deutschen bestehen, wenn relevante Einflussfaktoren kontrolliert werden (Baykara-Krumme 2008). Praktische Hilfe erbringen türkeistämmige Töchter häufiger, auch unter Kontrolle verschiedener Bedürfnisund Opportunitätsstrukturen (Schans/Komter 2010). Söhne unterscheiden sich dagegen nicht. Dieses Muster weicht zwar von dem traditionell-patrilinealen Modell ab, wonach Frauen nicht den eigenen Eltern, sondern primär den Schwiegereltern verpflichtet sind. Gegen das von den Autoren vorgebrachte Argument, dass es sich hier um ein migrationsspezifisches Phänomen handelt, spricht aber, dass die eigenen Eltern auch in der Türkei eine wichtigere Rolle im sozialen Netzwerk der Töchter einnehmen als die Schwiegereltern (Nauck/Suckow 2006). Finanzielle Transfers von Kindern schließlich sind selten, aber häufiger als in der deutschen Bevölkerung, Transfers an Kinder kommen signifikant seltener vor (Baykara-Krumme 2008b). In Frankreich zeigte sich, dass in muslimischen Migrantenfamilien (aus patrilinealen Herkunftskontexten) Söhne häufiger Transfers erhalten als Töchter, und Ältere tendenziell sowohl intergenerational auf- als auch abwärts 
mehr finanzielle Hilfe leisten als sie erhalten (Attias-Donfut/Wolff 2008). Dabei sind transnationale Beziehungen durch Rücküberweisungen ins Herkunftsland charakterisiert. Entscheidend sind hier die große Wohnentfernung, die andere Unterstützungsformen erschwert, aber auch die unterschiedlichen Kontexte mit ihren jeweiligen (finanziellen) Ressourcenstrukturen. Ratschläge und emotionale Hilfe sind vielfach nur möglich, wenn man die Situation, in der der andere sich befindet, kennt: Trotz der Entwicklung in der Kommunikationstechnologie und den vielen (günstigen) Möglichkeiten für transnationale Kontakte ohne physische Mobilität bleiben transnationale Beziehungen durch die Entfernung geprägt. So berichten Şenyürekli/Detzner (2008), dass in der Türkei lebende Eltern und in den USA wohnhafte Kindern über gewisse Themen bewusst nicht miteinander sprechen, um sich gegenseitig möglichst wenig zu belasten.

Nachdem eine gute familiäre Einbettung lange als migrantenspezifisches soziales Kapital (im Alter) galt, wird dies, auch vor dem Hintergrund intensivierter Forschung, zunehmend differenzierter gesehen (Zimmermann 2012). Über die Generationen scheint sich zumindest in den Werteeinstellungen zur familialen Solidarität wenig zu verändern (Carnie/Baykara-Krumme in diesem Band, siehe auch Arends-Toth/Van de Vijer 2008). Aber inwiefern unterscheiden sich Migranten bzw. ihre Nachkommen damit eigentlich von den ,peers“ im Herkunftsland?

\subsection{Hypothesen}

Zum Einfluss einer Migration auf intergenerationale Einstellungs- und Verhaltensmuster lassen sich auf Basis dieser Ausführungen gegensätzliche Hypothesen formulieren. So können neue Opportunitätsstrukturen mit entsprechend veränderten Ressourcen und $\mathrm{Be}$ dürfnissen sowie die individuellen und intergenerationalen Akkulturationsprozesse die Kontakthäufigkeit und kognitive Unterstützung gegenüber dem Herkunftskontext prinzipiell sowohl erhöhen (Hypothese 1) als auch schmälern (Hypothese 2) und - angesichts dieser komplexen Gemengelage - zu neuen ambivalenten und hybriden Formen führen. Für die finanzielle Unterstützung ist zunächst zu erwarten (Hypothese 3), dass ältere Migranten in Westeuropa von ihren Kindern seltener Transfers erhalten als Nichtmigranten in der Türkei, und sie ihre Kinder zugleich häufiger finanziell unterstützen, trotz ihres tendenziell hohen Armutsrisikos (Tucci/Yıldız 2012). Transnationale Beziehungen schließlich sind durch Transfers der in Westeuropa lebenden Kinder an die Eltern in die Türkei gekennzeichnet. Wird angenommen, dass sich Werte langsamer verändern als Verhaltensmuster (Nauck 1988), so sollten die Gruppenunterschiede in den Einstellungen entsprechend geringer sein (Hypothese 4).

Das Bildungsniveau und die Erwerbstätigkeit, strukturelle Indikatoren für veränderte Opportunitätsstrukturen und Akkulturationsbedingungen, werden als Prädiktor- und Mediatorvariablen berücksichtigt. Mit dem Bildungsniveau werden individualistische Einstellungen und das Ziel der individuellen Autonomie bedeutsamer. Die Alternativen zur (Herkunfts-) Familie, wie interethnische Freundschaftsnetzwerke, eine Erwerbstätigkeit oder die berufliche Karriere wachsen (Kalmijn 2006), zudem nehmen die Zeitressourcen ab. Die Generationensolidarität ist demnach bei höherer Bildung und bei Erwerbstätigkeit geringer (Hypothese 5). Die Wohnentfernung erhält im Migrationskontext besondere Bedeutung (Silverstein/Attias-Donfut 2010). Grundsätzlich sollte die Relevanz für die Gene- 
rationensolidarität hoch sein (Hypothese 6), allerdings mit geringen Differenzen zwischen Migranten- und Nichtmigrantenfamilien, sondern vielmehr mit einem vermittelnden Effekt für die transnationalen Familien. Als demographische Merkmale werden das Geschlecht und der Familienstand des Kindes, die Existenz von (Enkel-)Kindern, die Zahl der Geschwister sowie die Information, ob noch beide Eltern oder nur noch ein Elternteil lebt, berücksichtigt (dazu van Gaalen/Dykstra 2006; Steinbach/Kopp 2008, BaykaraKrumme et al. 2011).

\section{Datenbasis und Methode}

Als Datensatz dient der LineUp-Survey "2000 Families. Migration Histories of Turks in Europe", dessen Haupterhebungsphase 2011 in fünf Regionen in der Türkei stattfand, die von der Auswanderung im Rahmen der Gastarbeiteranwerbung zwischen 1960 und 1974 besonders betroffen waren. ${ }^{2}$ Basierend auf einem „random route“-Verfahren wurden Haushalte in den fünf Herkunftsregionen gescreent und auf diese Weise Familien ausgewählt, zu deren näherer oder weiterer Verwandtschaft ein ehemaliger Arbeitsmigrant zählt. Ausgehend von diesem männlichen Familienoberhaupt, das, so lautete das Selektionskriterium, heute zwischen 65 und 90 Jahre alt ist (bzw. so alt wäre, wenn es noch leben würde), in der Region aufgewachsen ist und zwischen 1960 und 1974 für mindestens fünf Jahre nach Europa ausgewandert ist, wurden Informationen über ihn, die Kinder, Enkel und Urenkel gesammelt, unabhängig von ihrem aktuellen Wohnort. Die Vergleichsgruppe der Nichtmigranten umfasst in jeder Region 20 Prozent des Samples. Dabei handelt es sich um gleichaltrige Männer mit ihren Nachkommen, die nicht nach Europa emigrierten. In jeder Region wurden etwa 400 Familien befragt, u.a. mit persönlichen, standardisierten, etwa halbstündigen Interviews mit zufällig ausgewählten Familienmitgliedern. Die folgenden Analysen stützen sich auf die Interviews mit den (bis zu zwei) zufällig ausgewählten Kindern der Zielpersonen, also den Kindern der nun 65- bis 90jährigen früheren Arbeitsmigranten und ihrer nichtmigrierten ,„peers“, die jeweils zu den Kontakten und Austauschbeziehungen mit ihren Eltern befragt wurden.

Die Nichtmigrantenfamilien $\left(\mathrm{n}=279^{3}\right)$ sind definiert als ,türkische Familien in der Türkei“, in denen der Vater nicht nach Europa emigrierte und die Mutter oder/und der Vater heute ausschließlich in der Türkei leben. Das befragte Kind ist in der Türkei geboren, hat dort den Schulabschluss gemacht und lebt ausschließlich dort. Die Migrantenfamilien ( $\mathrm{n}=329)$ dagegen sind definiert als „türkeistämmige Familien in Westeuropa“, in

2 Die Studie wird im Rahmen des NORFACE Research Programme Migration in Europe von 2009 bis 2013 finanziell gefördert. Sie widmet sich den Folgen der Gastarbeiteremigration aus der Türkei nach Europa. Die Leitung des Projekts liegt bei Dr. Ayşe Güveli (University of Essex), Prof. Harry Ganzeboom (Freie Universität Amsterdam) und Prof. Bernhard Nauck (Technische Universität Chemnitz). Die Herkunftsregionen sind Acıpayam (Provinz Denizli), Akçaabat (Provinz Trabzon), Emirdağ (Provinz Afyonkarahisar) und Kulu (Provinz Konya). In der Region Şarkışla (Provinz Sivas) wurde die Pilotstudie durchgeführt, deren Daten aufgrund von veränderten Items zu den Werteinstellungen im Folgenden allerdings nicht berücksichtigt werden.

3 Fälle ohne mindestens einen lebenden Elternteil (Mutter oder Vater) und mit fehlenden Werten in den hier interessierenden Ziel- und Einflussvariablen (letzteres implizierte nur sehr wenige zusätzliche Ausfälle) wurden aus der Analyse ausgeschlossen. 
denen der Vater als Arbeitsmigrant nach Westeuropa emigrierte und heute die Mutter oder/und der Vater in Westeuropa leben oder pendeln. Das Kind hat seinen Schulabschluss in Westeuropa gemacht und lebt heute dort. Von zentraler Bedeutung ist also, ob Eltern und Kinder eine Migrationserfahrung mit anschließendem Aufenthalt außerhalb des Herkunftslandes haben. Selbst dort, wo Familienangehörige pendeln, wird von einem nachhaltigen Einfluss des Zielkontexts ausgegangen. Ein anderes Interesse bestimmt die Auswahl transnationaler Familien ( $\mathrm{n}=79)$. Im Fokus steht die Transnationalität der Beziehung, die zwei nationale Kontexte mit unterschiedlichen Opportunitätsstrukturen umfasst, verbunden mit einer großen räumlichen Distanz. Als „transnationale Familien“ wurden jene Familien definiert, in denen die Eltern derzeit ausschließlich in der Türkei wohnhaft sind (unabhängig von einem früheren Aufenthalt in Westeuropa) und das Kind derzeit ausschließlich in Westeuropa lebt (in zwei Fällen handelt es sich um außereuropäische Länder). Damit grenzt sich diese Gruppe klar von den beiden anderen Gruppen ab. ${ }^{4}$

Abhängige Variablen: Basierend auf dem Modell der intergenerationalen Solidarität (vgl. Bengtson/Roberts 1991) stehen die Kontakthäufigkeit, die finanzielle und kognitive Hilfe sowie Werteinstellungen im Fokus. Die Werteinstellungen sind neben der Wohnentfernung zugleich eine unabhängige Variable. Die Informationen zu den Generationenbeziehungen beziehen sich im Fragebogen auf beide Elternteile, sie wurden nicht separat für Mütter und Väter erhoben. Wenn eines der beiden Elternteile verstorben war, so waren die Befragten aufgefordert, sich zu den Beziehungen zu dem lebenden Elternteil zu äußern. Für die Werteinstellungen wurde nach dem Grad der Zustimmung zu den folgenden Items gefragt: „Kinder sollten alle notwendigen Opfer erbringen, um sich um ihre pflegebedürftigen Eltern kümmern zu können“ und „Eltern sollten ihren Kindern finanziell helfen, selbst wenn sie sich dadurch verschulden würden". Die Antwortkategorien umfassen drei Items ( 1 ,stimme zu“ 2 ,weder noch“ 3 ,stimme nicht zu), für die im Falle einer Zustimmung und Ablehnung jeweils nach dem Grad gefragt wurde (1 „etwas“ bzw. 2 ,sehr stark"), so dass eine fünfstufige Antwortskala vorliegt. ${ }^{5}$ Die Kontakthäufigkeit bezieht sich auf alle Kontakte: „Wie oft haben Sie Kontakt (persönlich, telefonisch, über das Internet, Emails oder Briefe) mit Ihren Eltern/Ihrer Mutter/Ihrem Vater?“ Die Antwortkategorien reichen von 0 „täglich“, 1 „,nahezu täglich“, 2 „etwa einmal in der Woche“, 3 „etwa einmal im Monat“, 4 „mehrmals im Jahr“, 5 „,seltener“ bis zu 6 „,nie“. Kognitive Hilfe wurde wie folgt erhoben: „Wie oft haben Sie in den vergangenen 12 Monaten Ihren Eltern/Ihrer Mutter/Ihrem Vater mit Ratschlägen bei persönlichen Problemen zur Seite gestanden?“, und finanzielle Hilfe bezieht sich auf finanzielle Unterstützung oder umfangreiche Geld- oder Sachgeschenke an die Eltern/die Mutter/den Vater in den 12 Monaten vor dem Interview. Gleichlautende Fragen wurden in Hinblick auf Unterstützungsleistungen von den Eltern gestellt. Die Antwortitems sind jeweils 1 „nahezu täglich“, 2 „etwa

4 Wichtigste europäische Heimatländer der türkeistämmigen Kinder in den hier analysierten Migranten- und transnationalen Familien sind Deutschland (28\%) und Belgien (28\%), gefolgt von Frankreich (13\%), den Niederlanden (11\%) und Schweden (7\%). Insgesamt emigrierten von allen ehemaligen Arbeitsmigranten im Hauptsample des LineUp-Surveys 56\% nach Deutschland, 11\% nach Frankreich, 10\% nach Belgien, 7\% in die Niederlande, 7\% nach Österreich, $5 \%$ nach Dänemark, $3 \%$ nach Schweden und $1 \%$ in die Schweiz.

5 Die Wertitems korrelieren in dem hier betrachteten Subsample signifikant, aber doch vergleichsweise niedrig (,19, $\mathrm{p}<, 001$, Pearson), so dass sie getrennt berücksichtigt werden. 
einmal in der Woche“, 3 „etwa einmal im Monat“, 4 „,mehrmals im Jahr“, 5 „seltener“ und 6 ,nie“. Alle Skalen werden für die Analysen invertiert.

Unabhängige Variablen: Neben den Variablen des Migrationshintergrunds bzw. der Transnationalität der Beziehung werden demographische und strukturelle Merkmale des Kindes berücksichtigt, wie das Geschlecht $(1=$ Tochter, $0=$ Sohn $)$ und das Alter (metrische Variable). Das hier interessierende Bildungsniveau wird als metrische Variable operationalisiert. Erhoben wurde der höchste Bildungsabschluss anhand einer Skala, in der die europäischen länderspezifischen Bildungsabschlüsse den in der Türkei existierenden Bildungsabschlüssen zugeordnet waren, so dass sich die Befragten selbst dem türkischen Äquivalent zuordnen konnten. Für die Analysen wird die zugrunde liegende türkische Matrix verwendet. ${ }^{6}$ Personen, die zum Zeitpunkt der Befragung erwerbstätig waren, sich in Aus- oder Weiterbildung befanden oder anderweitig unentgeltlich tätig waren, werden mit jenen, die nicht außerhalb des Haushalts tätig waren (Hausarbeit, Krankheit, Arbeitslosigkeit), verglichen. Von Bedeutung sind hier primär die zeitlichen Ressourcen sowie die Opportunitäten für außerfamiliale Kontakte durch die Tätigkeit. Für den Familienstand ,verheiratet" werden Verheiratete und in eingetragener Partnerschaft lebende Personen zusammengefasst. Da die Zahl der Kinderlosen relativ gering ist, werden Personen ohne Kinder und mit höchstens einem Kind zusammengefasst (0) und der Gruppe der Personen mit mindestens zwei Kindern gegenübergestellt (1). Differenziert wird des Weiteren danach, welche Elternteile noch leben, sowie nach der Anzahl der Kinder, die die Eltern insgesamt haben. Als Merkmale der Beziehung gesondert berücksichtigt werden die Werteinstellungen (siehe oben) sowie die Wohnentfernung. Hier wurde erhoben, wie lange es zeitlich dauert, zu den Eltern/der Mutter/dem Vater zu gelangen, mit 0 ,gleicher Haushalt", 1 „weniger als 10 Minuten“, 2 „,10 bis 30 Minuten“, 3 „30 bis 60 Minuten“, 4 „1 bis 3 Stunden“, 5 „,mehr als drei Stunden, weniger als ein Tag“, 6 „mehr als ein Tag“. Tabelle 1 zeigt die Verteilung der Prädiktoren in den drei hier interessierenden Gruppen.

6 Die Kategorien lauten: 1 „ohne Schulabschluss“, 2 „Abschluss Grundschule, 5 Jahre“, 3 „Abschluss Grundschule, 8 Jahre“, 4 „Allgemeiner mittlerer Schulabschluss“, 5 „Beruflicher mittlerer Schulabschluss“, 6 „Allgemeiner höherer Schulabschluss/Abitur“, 7 „Beruflicher höherer Schulabschluss“, 8 „Abschluss Fach(hoch)schule“, 9 „Abschluss Universität, Bachelor“, 10 „Abschluss Universität, Master“, 11 „Abschluss Promotion“. 
Tabelle 1: Deskription der Zielvariablen und Prädiktoren

\begin{tabular}{|c|c|c|c|}
\hline & $\begin{array}{l}\text { Familien } \\
\text { in der Türkei }\end{array}$ & Migrantenfamilien & $\begin{array}{l}\text { Transnationale } \\
\text { Familien }\end{array}$ \\
\hline \multicolumn{4}{|l|}{ Zielvariablen } \\
\hline $\begin{array}{c}\text { Kinder sollen Eltern im Pflegefall helfen } \\
1 \text { „stimme überhaupt nicht zu“ - } \\
5 \text { „stimme voll und ganz zu“ }\end{array}$ & $4,7(0,7)$ & $4,6(0,9)$ & $4,7(0,7)$ \\
\hline $\begin{array}{l}\text { Eltern sollen Kindern finanziell helfen } \\
1 \text {,stimme überhaupt nicht zu“ - } \\
5 \text { „stimme voll und ganz zu“ }\end{array}$ & $4,1(1,2)$ & $3,9(1,3)$ & $4,1(1,2)$ \\
\hline $\begin{array}{l}\text { Kontakthäufigkeit } \\
1 \text { „nie“ }-7 \text { „täglich“ }\end{array}$ & $5,8(1,2)$ & $6,0(1,1)$ & $5,4(1,1)$ \\
\hline $\begin{array}{l}\text { Rat an Eltern } \\
\qquad 1 \text { „nie“ }-6 \text { „nahezu täglich“ }\end{array}$ & $3,9(2,0)$ & $4,1(1,8)$ & $3,9(1,6)$ \\
\hline $\begin{array}{l}\text { Rat von Eltern } \\
\qquad 1 \text { „nie“ }-6 \text { „nahezu täglich“ }\end{array}$ & $3,8(2,0)$ & $3,9(1,9)$ & $3,7(1,7)$ \\
\hline $\begin{array}{l}\text { Transfers an Eltern } \\
1 \text { „nie“ }-6 \text { „nahezu täglich“ }\end{array}$ & $2,3(1,5)$ & $2,6(1,5)$ & $2,2(1,0)$ \\
\hline $\begin{array}{l}\text { Transfers von Eltern } \\
1, \text { nie“ }-6 \text {,nahezu täglich“ } \\
\end{array}$ & $2,0(1,3)$ & $2,1(1,4)$ & $1,5(0,8)$ \\
\hline \multicolumn{4}{|c|}{ Prädiktoren: Demographisch-strukturelle Merkmale des Kindes } \\
\hline Tochter & $51,2 \%$ & $43,5 \%$ & $39,2 \%$ \\
\hline Alter (Jahre) & $41(8,8)$ & $38(7,5)$ & $43(7,5)$ \\
\hline $\begin{array}{l}\text { Höchster Bildungsabschluss } \\
1 \text { „ohne Schulabschluss“ - } 11 \text { „Pror }\end{array}$ & $3,9(2,6)$ & $6,3(1,8)$ & $4,1(2,3)$ \\
\hline Erwerbstätig/in Ausbildung & $57,9 \%$ & $75,7 \%$ & $64,6 \%$ \\
\hline Verheiratet/mit Partner & $86,9 \%$ & $85,1 \%$ & $93,7 \%$ \\
\hline Mindestens 2 Kinder & $76,8 \%$ & $74,3 \%$ & $89,9 \%$ \\
\hline \multicolumn{4}{|c|}{ Prädiktoren: Demographisch-strukturelle Merkmale der Familie } \\
\hline Beide Eltern leben & $63,2 \%$ & $80,3 \%$ & $63,2 \%$ \\
\hline Nur Mutter lebt & $25,3 \%$ & $17,0 \%$ & $31,7 \%$ \\
\hline Nur Vater lebt & $11,5 \%$ & $2,7 \%$ & $5,1 \%$ \\
\hline Anzahl der Geschwister & $5,5(2,2)$ & $4,9(1,6)$ & $5,7(2,1)$ \\
\hline Wohnentfernung & $3,4(1,7)$ & $3,0(1,8)$ & $6,2(, 06)$ \\
\hline$\underline{n} \quad 0$ "gleicher Haushalt" -6 „,mehr al & 297 & 329 & 79 \\
\hline
\end{tabular}

Datenbasis: LineUp-Survey 2011. Angegeben sind, soweit nicht anders vermerkt, die Mittelwerte und Standardabweichungen.

Die Kinder in Migrantenfamilien haben ein höheres Bildungsniveau erreicht und sind häufiger erwerbstätig als jene in Nichtmigrantenfamilien in der Türkei. Diese Verteilung hängt vermutlich mit dem geringeren Frauenanteil in den Migrantenfamilien dieser (Teil-) Stichprobe zusammen. Bei Migrantenfamilien ist die Wohnentfernung deutlich geringer, es leben noch häufiger beide Elternteile, und die Zahl der Geschwister ist kleiner. Transnationale Familien sind vor allem durch die große Wohnentfernung charakterisiert. Hier ist der Anteil der Söhne besonders hoch.

Berechnet wurden verschiedene lineare Regressionsmodelle (OLS). Für jede Zielvariable wird das vollständige Modell mit den Beta-Koeffizienten präsentiert, das alle hier zu berücksichtigenden Prädiktoren enthält. Im Text wird jeweils auch auf die Befunde der (nicht dargestellten) Stufenmodelle eingegangen. Da aufgrund des Familiendesigns bis zu 
zwei Kinder einer Familie Angaben zu einem Elternteil bzw. denselben Eltern gemacht haben, also keine Unabhängigkeit vorliegt, wurde zur Korrektur der Standardfehler für die Familiencluster kontrolliert.

\section{Empirische Befunde}

\subsection{Deskriptive Ergebnisse}

Tabelle 1 zeigt die Ergebnisse für die Zielvariablen. Insgesamt sind die Generationenbeziehungen durch eine hohe normative Solidarität sowie eine ausgeprägte Kontakthäufigkeit charakterisiert. Kognitive Unterstützung wird ebenfalls relativ häufig geleistet, während Transfers eher selten sind. Die befragten Kinder tendieren dazu, die von ihnen erbrachten Leistungen häufiger zu nennen als diejenigen der Eltern an sie und bewerten zugleich die Hilfe von Kindern an Eltern als wichtiger: Sowohl normativ als auch faktisch zeigen sich die Kinder ihren Eltern solidarisch verpflichtet. Im Unterschied zu Familien in der Türkei sind die Generationenbeziehungen in Migrantenfamilien in Westeuropa v.a. durch eine höhere Kontakthäufigkeit $(6,0 \mathrm{zu} 5,8, \mathrm{p}<, 05)$ und, entgegen den Erwartungen, häufigere finanzielle Transfers der Kinder an die Eltern gekennzeichnet $(2,6 \mathrm{zu}$ 2,3, $\mathrm{p}<, 05)$. Kinder helfen ihren Eltern in der Migration auch häufiger mit Ratschlägen, allerdings sind diese Differenzen, wie auch die übrigen, nicht signifikant. Damit sind die Generationenbeziehungen in der Migration tendenziell intensiver und von häufigerer Unterstützung geprägt als im Herkunftsland, während gleichzeitig, und das ist bemerkenswert, die Werteinstellungen hinsichtlich gegenseitiger Unterstützungsverpflichtungen bei den Kindern in der Migration geringer ausgeprägt sind als im Herkunftsland (jeweils auf einem Signifikanzniveau von $p<, 05$ ). Transnationale Familien unterscheiden sich auf zwei Dimensionen deutlich von den Familien in der Türkei: Die Kontakthäufigkeit ist deutlicher geringer $(5,4, p<, 01)$ und Transfers von Eltern (in der Türkei) an Kinder (in Westeuropa) sind signifikant seltener $(1,5, \mathrm{p}<, 001)$. Entgegen den Erwartungen finden sich jedoch keine signifikanten Differenzen bei den Transfers der Kinder an die Eltern.

\subsection{Multivariate Modelle}

Es soll nun geprüft werden, worauf die beobachteten Differenzen zwischen den drei Gruppen zurückzuführen sind, ob es sich tatsächlich um direkte Migrationseffekte handelt oder nicht vielmehr um Kompositionseffekte bzw. indirekte Migrationseffekte, z.B. im Sinne einer Mediation (oder Suppression) durch kulturelle, demographische und soziostrukturelle Merkmale.

Werteinstellungen: Im Modell zur Analyse der Werteinstellung hinsichtlich der Pflege der alten Eltern durch die Kinder lassen sich bivariat die oben beschriebenen Ergebnisse für Migranten im Vergleich zu Kindern im Herkunftsland bestätigen $(-, 32, p<, 10)$. Unter Kontrolle von Geschlecht, Alter, Bildung und Erwerbsstatus sinkt der Koeffizient bereits deutlich, das Signifikanzniveau steigt $(-, 15, \mathrm{p}<, 05)$. Die deutlich höhere Bildung und die häufigere Erwerbstätigkeit unter Migranten können die Unterschiede allein nicht erklären. 
Weder das Bildungsniveau noch der Erwerbsstatus haben einen eigenständigen signifikanten Einfluss. Erst bei Berücksichtigung weiterer Merkmale wird der Effekt des Migrationsstatus insignifikant, der Koeffizient bleibt jedoch bemerkenswert hoch und ändert sein Vorzeichen nicht (-,11, siehe Tabelle 2). Möglicherweise handelt es sich hier um einen Akkulturationseffekt, der auf den spezifischen westeuropäischen Kontext mit seinem Angebot an staatlichen Hilfen zurückzuführen ist: Eine gute Versorgung der Eltern ist auch ohne allzu große Opfer der Kinder möglich. Als einflussreiche Prädiktoren erweisen sich demographische und familiale Merkmale: Kinder, die selbst mindestens zwei Kinder haben, stimmen der Aussage signifikant seltener zu. (Antizipierte) Zeitressourcen können hier eine Rolle spielen, aber auch der mögliche Gedanke, den eigenen Kindern (später) nicht zur Last fallen zu wollen. Zugleich ist in den Familien, in denen die Mutter verstorben ist und allein der Vater noch lebt, die Wahrscheinlichkeit für eine Zustimmung größer. Hier liegt eine solche Bedarfssituation möglicherweise bereits vor, ohne die Generationensolidaritätspotenziale zu schmälern. Insgesamt bleibt die Erklärungskraft der Modelle allerdings sehr gering.

Tabelle 2: Regressionsmodelle zu Werteinstellungen (Beta-Koeffizienten)

\begin{tabular}{|c|c|c|}
\hline & $\begin{array}{l}\text { Pflege der Eltern } \\
\text { durch Kinder }\end{array}$ & $\begin{array}{c}\text { Transfers der Eltern } \\
\text { an Kinder }\end{array}$ \\
\hline Migrantenfamilien & $-0,11(0,07)$ & $-0,10(0,11)$ \\
\hline Transnationale Familien & $0,05(0,10)$ & $0,07(0,17)$ \\
\hline \multicolumn{3}{|l|}{ (Ref.: Familien in der Türkei) } \\
\hline Tochter & $-0,01(0,07)$ & $-0,11(0,11)$ \\
\hline Alter & $0,00(0,00)$ & $0,01(0,01)^{\circ}$ \\
\hline Höchster Bildungsabschluss & $0,01(0,01)$ & $-0,05(0,02)^{\circ}$ \\
\hline Erwerbstätig/in Ausbildung & $-0,07(0,07)$ & $-0,20(0,11)^{\circ}$ \\
\hline Verheiratet/mit Partner & $0,05(0,08)$ & $0,01(0,17)$ \\
\hline Mindestens 2 Kinder & $-0,20(0,07)^{\star *}$ & $-0,18(0,12)$ \\
\hline Nur Vater lebt & $0,22(0,07)^{\star *}$ & $0,11(0,20)$ \\
\hline Nur Mutter lebt & $0,05(0,07)$ & $0,04(0,11)$ \\
\hline \multicolumn{3}{|l|}{ (Ref.: Beide Eltern leben) } \\
\hline Anzahl der Geschwister & $0,01(0,01)$ & $-0,04(0,03)$ \\
\hline Wohnentfernung &,$- 02(0,02)$ & $-0,05(0,03)^{\circ}$ \\
\hline Konstante & $4,74(0,22)^{\star * *}$ & $4,50(0,35)^{\star * *}$ \\
\hline$n$ & 705 & 700 \\
\hline korrigiertes $r^{2}$ & 0,02 & 0,04 \\
\hline
\end{tabular}

Datenbasis: LineUp-Survey 2011, Signifikanzniveaus: ${ }^{\circ} \mathrm{p}<, 10, * \mathrm{p}<, 05, * * \mathrm{p}<, 01, * * * \mathrm{p}<, 001$, kontrolliert für Familiencluster.

Dies gilt auch für das Modell zur Norm intergenerational abwärts gerichteter Hilfeleistungen. Hier finden sich bivariat die oben genannten Unterschiede zwischen Migranten und Kindern in der Türkei $(-, 24, p<, 05)$. Bei Kontrolle weiterer demographischer und soziostruktureller Merkmale verschwinden diese Unterschiede, ohne dass im Gesamtmodell der Koeffizient wirklich gegen Null tendiert. Sowohl dem Bildungsniveau als auch dem Erwerbsstatus kommt eine wichtige Bedeutung als Mediator zu, beide zeigen zudem einen eigenständigen negativen Effekt im Gesamtmodell. Mit größerer (finanzieller) Unabhängigkeit sinkt die Erwartung an elterliche Unterstützung, was vermutlich weniger als Zeichen verringerter Generationensolidarität in der Migration zu interpretieren ist, denn 
als Ausdruck von geringem Bedarf und möglicherweise dem Wunsch, den Eltern nicht zur Last zu fallen. Während Unterstützungsnormen also in der Migration tendenziell auch bei Kontrolle anderer Faktoren weniger Zustimmung erfahren als in der Türkei und daher als „Migrationseffekte“ interpretiert werden können, unterscheiden sich transnationale Familien von nichtmigrierten Familien nicht.

Kontakthäufigkeit und kognitive Unterstützung: Dies ist völlig anders bei der Kontakthäufigkeit. Während die bivariaten Differenzen zwischen Migranten und Nichtmigranten $(, 25, \mathrm{p}<, 01)$ bei Kontrolle anderer Variablen nicht mehr signifikant sind, also durch diese erklärt werden können, sind Unterschiede zwischen transnationalen Familien und Nichtmigranten in der Türkei genuin mit der Transnationalität der Familie verbunden. Bemerkenswert ist, wie zu erwarten, der Einfluss der Wohnentfernung. Ohne Kontrolle dieses strukturellen Merkmals ist die Wahrscheinlichkeit häufigen Kontakts in transnationalen Familien sehr viel geringer (Gesamtmodell: -,45, $\mathrm{p}<, 01$ ). Kontrolliert man für diese Variable im Modell, so ist der Effekt signifikant positiv (siehe Tabelle 3). Im Vergleich zu den anderen (wenigen) Familien, deren Mitglieder genauso weit voneinander entfernt wohnen, ist die Wahrscheinlichkeit häufigen Kontakts in transnationalen Familien demnach sogar größer. Das in einer großen Wohnentfernung implizierte Entfremdungspotenzial ist im Fall transnationaler türkischer Familien also gering, selbst wenn die größere Wohnentfernung die Kontakthäufigkeit faktisch verringert.

Tabelle 3: Regressionsmodelle zu Kontakt und kognitiver Unterstützung (Beta-Koeff.)

\begin{tabular}{|c|c|c|c|}
\hline & Kontakthäufigkeit & Unterstützung an Eltern & Unterstützung von Eltern \\
\hline Migrantenfamilien & $0,01(0,09)$ & $-0,03(0,17)$ & $-0,04(0,18)$ \\
\hline $\begin{array}{l}\text { Transnationale Familien } \\
\text { (Ref.: Familien in der Türkei) }\end{array}$ & $0,33(0,16)^{*}$ & $0,47(0,26)+$ & $0,44(0,26)^{\circ}$ \\
\hline Tochter & $-0,07(0,08)$ & $0,49(0,15)^{\star *}$ & $0,06(0,17)$ \\
\hline Alter & $-0,00(0,01)$ & $0,02(0,01)^{\circ}$ & $0,00(0,01)$ \\
\hline Höchster Bildungsabschluss & $0,03(0,02)^{\circ}$ & $0,08(0,03)^{* *}$ & $0,01(0,04)$ \\
\hline Erwerbstätig/in Ausbildung & $-0,09(0,09)$ & $0,20(0,17)$ & $-0,04(0,17)$ \\
\hline Verheiratet/mit Partner & $0,11(0,13)$ & $-0,00(0,22)$ & $-0,39(0,23)^{\circ}$ \\
\hline Mindestens 2 Kinder & $0,12(0,10)$ & $0,01(0,19)$ & $-0,05(0,21)$ \\
\hline Nur Vater lebt & $-0,26(0,16)$ & $-0,05(0,27)$ & $-0,16(0,27)$ \\
\hline $\begin{array}{l}\text { Nur Mutter lebt } \\
\text { (Ref.: Beide Eltern leben) }\end{array}$ & $0,04(0,09)$ & $0,20(0,18)$ & $-0,08(0,20)$ \\
\hline Geschwister & $-0,07(0,02)^{\star \star}$ & $-0,08(0,04)^{\star}$ & $-0,13(0,04)^{\star *}$ \\
\hline Einstellung: Hilfe an Eltern & $0,12(0,06)^{*}$ & $0,29(0,10)^{* *}$ & $0,31(0,10)^{* *}$ \\
\hline Wohnentfernung & $-0,28(0,03)^{* * *}$ & $-0,21(0,04)^{* * *}$ & $-0,17(0,04)^{* * *}$ \\
\hline Konstante & $6,48(0,04)^{* * *}$ & $2,19(0,10)^{* *}$ & $3,92(0,72)^{* * *}$ \\
\hline$n$ & 700 & 700 & 699 \\
\hline korrigiertes $r^{2}$ & 0,26 & 0,08 & 0,07 \\
\hline
\end{tabular}

Datenbasis: LineUp-Survey 2011, Signifikanzniveaus: ${ }^{\circ} \mathrm{p}<, 10,{ }^{*} \mathrm{p}<, 05, * * \mathrm{p}<, 01,{ }^{* * *} \mathrm{p}<, 001$, kontrolliert für Familiencluster.

Bei der kognitiven Generationensolidarität sind die Differenzen nach Migrationshintergrund bereits im Ausgangsmodell gering (Tabelle 1). In den multivariaten Analysen (Tabelle 3) zeigen sich keine signifikanten Migrationseffekte, auch nicht im Sinne von Suppression. Erneut erweist sich die Wohnentfernung als eine Opportunität für transnationale 
Familien. Unter Kontrolle dieser Gelegenheitsstruktur ist kognitive Hilfe an und von Eltern in transnationalen Familien signifikant wahrscheinlicher als bei Nichtmigranten, ohne Kontrolle ist der Effekt jeweils nur leicht negativ und insignifikant (nicht dargestellt), was die Bedeutung der faktischen Generationensolidarität unter transnationalen Bedingungen unterstreicht. Hilfe an Eltern wird mit deutlich größerer Wahrscheinlichkeit von Töchtern geleistet. Dieser Geschlechtereffekt ist in Migrantenfamilien stärker ausgeprägt als bei Nichtmigranten im Herkunftsland (Interaktionseffekt: ,94, p<,01) und unterstützt damit die Befunde von Schans und Komter (2010). Für die Geschwister zeigen die Befunde den in der Literatur beschriebenen Substitutionseffekt (Steinbach/Kopp 2008): Je mehr Geschwister vorhanden sind, desto seltener wird von dem einzelnen Kind Hilfe geleistet. Entgegen den Annahmen zum Bildungsniveau (Hypothese 5) findet sich sowohl für die Kontakthäufigkeit als auch für die Hilfe an Eltern ein signifikant positiver Zusammenhang mit der Bildung. Offenbar führt eine höhere Bildung keineswegs zu einer Abwendung von der Familie. Vielmehr steigen mit den größeren Kompetenzen und dem zusätzlichen Wissen nicht nur die Möglichkeiten, sondern auch die Bereitschaft, den Eltern beratend zur Seite zu stehen. Dieser Mechanismus gilt für Migranten und Nichtmigranten in gleicher Weise (Interaktionseffekt ist insignifikant). Eine große Bedeutung hat in allen drei Modellen die Einstellung zur Hilfe an die Eltern. Dies zeigt, dass Werteinstellungen eindeutig positiv mit dem Verhalten zusammenhängen, wobei die kausale Richtung dieser Korrelation hier nicht eruiert werden kann.

Finanzielle Unterstützung: Entgegen der Hypothese 3 zeigte sich in Tabelle 1 bereits eine größere Häufigkeit finanzieller Unterstützung von Kindern an Eltern in Migrantenfamilien. Dieser Effekt (im bivariaten Modell: ,26, p<,05) kann jedoch mit differierenden demographischen Merkmalen der Kinder sowie deren Ressourcen nahezu komplett erklärt werden, ist also kein eigenständiger Migrations-, sondern vorrangig ein Kompositionseffekt. (Inwiefern die Merkmale der Kinder mit den Bedürfnissen der Eltern interagieren, wäre weitergehend zu überprüfen.) Generell unterstützen Töchter ihre Eltern seltener finanziell, sowohl in Migranten- als auch in Nichtmigrantenfamilien (Interaktionseffekt ist nicht signifikant). Anders als bei anderen Solidaritätsdimensionen zeigt sich hier kein signifikanter Effekt der Werteinstellung: Eine große Zustimmung zur Norm der Hilfe an pflegebedürftige Eltern bedeutet nur tendenziell eine größere Wahrscheinlichkeit häufigerer finanzieller Leistungen, obwohl es in beiden Fällen um „,bottom-up“-Hilfe geht. In transnationalen Familien sind - ohne Kontrolle der Wohnentfernung - finanzielle Hilfen der Kinder an die Eltern in der Türkei seltener (-,17, n.s.). Erst im Gesamtmodell, unter Berücksichtigung der Wohnentfernung, wird das Vorzeichen, wie in den vorgehenden Modellen, positiv. 
Tabelle 4: Regressionsmodelle zur finanziellen Unterstützung (Beta-Koeffizienten)

\begin{tabular}{|c|c|c|}
\hline & Kinder an Eltern & Eltern an Kinder \\
\hline Migrantenfamilien & $0,07(0,13)$ & $-0,02(0,12)$ \\
\hline Transnationale Familien & $0,33(0,16)^{*}$ & $0,05(0,15)$ \\
\hline \multicolumn{3}{|l|}{ (Ref.: Familien in der Türkei) } \\
\hline Tochter & $-0,63(0,12)^{\star \star \star}$ & $-0,14(0,11)$ \\
\hline Alter & $-0,01(0,01)$ & $-0,01(0,01)^{\circ}$ \\
\hline Höchster Bildungsabschluss & $-0,00(0,02)$ & $0,00(0,02)$ \\
\hline Erwerbstätig/in Ausbildung & $0,14(0,13)$ & $-0,30(0,13)^{\star}$ \\
\hline Verheiratet/mit Partner & $-0,28(0,19)$ & $-0,49(0,19)^{* *}$ \\
\hline Mindestens 2 Kinder & $0,03(0,14)$ & $-0,04(0,14)$ \\
\hline Nur Vater lebt & $0,05(0,22)$ & $0,11(0,18)$ \\
\hline Nur Mutter lebt & $0,05(0,13)$ & $-0,30(0,12)^{\star}$ \\
\hline \multicolumn{3}{|l|}{ (Ref.: Beide Eltern leben) } \\
\hline Anzahl der Geschwister & $-0,04(0,03)$ & $-0,08(0,03)^{\star *}$ \\
\hline Einstellung: Hilfe an Eltern & $0,07(0,07)$ & $0,15(0,05)^{\star *}$ \\
\hline Wohnentfernung & $-0,19(0,03)^{\star \star \star}$ & $-0,16(0,03)^{* * *}$ \\
\hline Konstante & $3,67(0,50)^{* * *}$ & $3,61(0,43)^{* * *}$ \\
\hline$n$ & 699 & 697 \\
\hline korrigiertes $r^{2}$ & 0,14 & 0,14 \\
\hline
\end{tabular}

Datenbasis: LineUp-Survey 2011, Signifikanzniveaus: ${ }^{\circ} \mathrm{p}<, 10,{ }^{*} \mathrm{p}<, 05,{ }^{* *} \mathrm{p}<, 01,{ }^{* * *} \mathrm{p}<, 001$, kontrolliert für Familiencluster.

Transfers von den Eltern sind sowohl in Migrantenfamilien als auch in Familien in der Türkei eher selten. Besonders selten sind sie, wie erwartet, im Fall transnationaler Familien (Hypothese 3). Ohne Kontrolle der Wohnentfernung ist der Effekt signifikant negativ $(-, 49, \mathrm{p}<, 001)$, erst unter Berücksichtigung dieses, die transnationalen Familien charakterisierenden Merkmals, verringert sich der Einfluss. Die Ressourcenstrukturen der Eltern (alleinstehende Mütter) und die Bedürfnisstrukturen der Kinder spielen eine zentrale Rolle (Alter, Erwerbsstatus, Familienstand). Werteinstellungen des Kindes haben einen signifikant positiven Einfluss, wenn es um die Hilfe der Eltern an die Kinder geht (Tabelle 4). Dies spricht wiederum für eine starke Kopplung von familialen Verhaltensmustern und individuellen Werteinstellungen, die ein reziprokes Moment beinhalten. Die Kinder, die die Norm stark unterstützen, erhalten mit größerer Wahrscheinlichkeit häufiger finanzielle Hilfe von den Eltern. Der Kausalität dieses Zusammenhangs kann hier nicht nachgegangen werden.

\section{Diskussion}

Seit vielen Jahren beschäftigt sich die sozialwissenschaftliche Forschung mit der Frage nach den Auswirkungen einer internationalen Migration auf die Ausgestaltung der familialen Generationenbeziehungen (Nauck 1997), zunehmend auch im Kontext der Lebensphase Alter (Baykara-Krumme 2008; de Valk/Schans 2008, Silverstein/Attias-Donfut 2010). Die hier verwendeten Daten erlauben eine neue Perspektive: Ausgehend vom Herkunftsland der Eltern dienen nichtgewanderte, also im Herkunftsland verbliebene sowie transnationale Familien als Vergleichsgruppe. Gegenüber der üblichen „Ziellandperspektive“ ermöglicht diese „Herkunftslandperspektive“ eine differenziertere Analyse der Mig- 
rationseffekte unter Kontrolle der kulturellen Herkunft, und erst mit einer Berücksichtigung der transnationalen Familiennetzwerke können Spezifika von Generationenbeziehungen unter Migrationsbedingungen umfassend aufgezeigt werden. Die Ergebnisse zeigen einige Unterschiede in den Verhaltensmustern, allerdings geringer und zum Teil in eine andere Richtung gehend als erwartet. Tendenziell scheinen sich die Generationenbeziehungen im Migrationskontext zu intensivieren (Hypothese 1): So ist die Kontakthäufigkeit bei Migranten größer als bei Nichtmigranten in der Türkei, und Kinder aus Migrantenfamilien unterstützen ihre Eltern häufiger finanziell. Die Befunde zu kognitiver Hilfe zeigen ebenfalls in diese Richtung, sind aber statistisch nicht signifikant. Die größere Häufigkeit von intergenerational aufwärts gerichteten Transfers in der Migration ist durchaus erstaunlich (und im Widerspruch zu Hypothese 3). Sind ältere Migranten in Westeuropa stärker bedürftig als Ältere im Herkunftsland? Ohne Berücksichtigung der Ressourcen der Älteren und vor dem Hintergrund, dass die Häufigkeit von Transfers wenig über ihren jeweiligen Umfang aussagt, lässt sich über die Bedeutung der prekären Lebensumstände vieler älterer Migranten in Westeuropa für die Hilfe durch die Kinder wenig sagen. Die vorgestellten Analysen unterstützen stärker das Argument von Kompositionseffekten in der Kindergeneration, die allerdings mit - unbeobachteten - strukturellen Merkmalen der Eltern korrespondieren können. Wie bei den anderen Beziehungsdimensionen bleiben bei den Transfers nach Berücksichtigung demographischer und struktureller Merkmale der Kinder keine Migrationseffekte bestehen, bei insgesamt sehr geringerem Einfluss von Bildungsniveau und Erwerbsstatus.

In den Einstellungsmustern finden sich ebenfalls geringe, aber insgesamt deutlichere Unterschiede zwischen Migranten in Westeuropa und Nichtmigranten in der Türkei, zumal diese auch nach Kontrolle weiterer Merkmale weitgehend (wenn auch statistisch nicht signifikant) bestehen bleiben. Diese Befunde sprechen gegen die Annahme, dass sich Werte langsamer verändern als das Verhalten (Hypothese 4), im Gegenteil. Hier kann es sich durchaus um Akkulturationseffekte in der Migration handeln, ohne dass die Generationensolidarität dadurch beeinträchtigt wäre: Ein „Wandel“ in den Einstellungen bei gleichzeitiger Kontinuität im Verhalten stellt eine spezifische Form der Akkulturation dar. Er gefährdet die Generationensolidarität selbst nicht. Denn das Angebot staatlicher Hilfen in den westeuropäischen Wohlfahrtsregimen ermöglicht eine umfassendere Unterstützung der Familie im Pflegefall Älterer als in der Türkei, so dass die notwendigen „Opfer“ seitens der Kinder geringer sein können, ohne dass die Älteren weniger oder schlechter versorgt wären. Alternative Unterstützungsstrukturen zur Familie existieren auch für erwachsene Kinder. Es ist weniger notwendig, dass sich Eltern für Kinder verschulden, um deren Wohlergehen zu sichern. In den Einstellungsmustern finden sich damit Hinweise auf ein „crowding out" in der Migration, ohne dass allerdings gleichzeitige Prozesse eines „crowding in“ auszuschließen sind (Künemund/Vogel 2006). Möglicherweise handelt es sich bei den geringeren Erwartungen an finanzielle Hilfe durch die Eltern auch um eine situative Reaktion angesichts deren geringer Ressourcen, denn immerhin unterstützen Migrantenkinder ihre Eltern finanziell häufiger. Umgekehrt ist die größere Zustimmung zu dieser Norm bei Kindern in der Türkei insofern bemerkenswert und Indikator für Wandel, als dass finanzielle Transfers von Eltern an erwachsene Kinder nicht den traditionellen Vorstellungen intergenerationaler Solidarität entsprechen (Ataca et al. 2005). Insgesamt unterstreicht die große Zustimmung zu beiden Normen sowohl in der Türkei als auch in Westeuropa die (andauernd) große Bedeutung der intergenerationalen gegenseiti- 
gen Solidaritätspotenziale. Generell sind Werteinstellungen ein abstraktes Maß, das nicht notwendigerweise mit tatsächlichen Handlungen übereinstimmen muss (Gans/Silverstein 2006). Nach den vorliegenden Daten sind Werteinstellungen allerdings ein zentraler Einflussfaktor, der mit den Verhaltensmustern stark korreliert. Transnationale Familien weisen im Vergleich der drei Gruppen die geringste Generationensolidarität auf, wobei sich dieser Befund in den multivariaten Modellen deutlich relativiert. Ausschlaggebend für die geringe Kontakthäufigkeit und Unterstützung ist, wie in Hypothese 6 postuliert, primär die Wohnentfernung, selbst wenn die hier untersuchten Dimensionen keine räumliche Nähe voraussetzen. Wird für den geographischen Faktor kontrolliert, so erweisen sich transnationale Familien als einander besonders nah: Das Entfremdungspotenzial ist hier vergleichsweise gering.

Bei aller Innovation des Designs der LineUp-Studie durch die Einbeziehung der nicht migrierten Bevölkerung im Herkunftsland, finden sich gleichwohl Limitationen. Erstens wurden in Hinblick auf die Generationenbeziehungen im Fragebogen nur wenige Items berücksichtigt. Das Thema umfasst aber viele weitere Aspekte, etwa die Motivationen, Machtbeziehungen und Ambivalenzen, die im Migrationskontext besonders relevant sein können, hier aber, wie so oft, unberücksichtigt bleiben müssen (Silverstein/Attias-Donfut 2010). Zweitens kann die grundsätzliche Frage, ob sich in der Migration zuerst das Verhalten oder die Einstellungen ändern, anhand der vorliegenden Querschnittsdaten nicht beantwortet werden (Nauck 1988). Dazu bedarf es individueller Paneldaten, die die Phasen vor und nach der Migration einschließen. Ein Vorteil der Daten ist grundsätzlich ihr multigenerationales, familienbasiertes Design. So ist eine Antwort auf die migrationssoziologisch wichtige Frage möglich, ob die Differenzen zwischen Migranten und Nichtmigranten im Herkunftsland, wie in der klassischen Assimilationsforschung postuliert, in der dritten (und vierten) Generation noch größer werden (Park 1964). Allerdings können, dies sei als dritte Limitation der Daten angeführt, Alterseffekte in diesem Design nicht von Kohorteneffekten getrennt werden (Bengtson 1975). Grundsätzlich sind größere Differenzen in nachfolgenden Generationen keinesfalls wahrscheinlich. Denn auch die Vergleichsgruppe im Herkunftsland hat über die vergangenen Jahre Wandel erlebt (Nauck/ Klaus 2005). Die Situation in der Türkei ist heute nicht mehr jene, die zum Zeitpunkt der Auswanderung vorherrschte. So lassen die Daten auch offen, ob nicht Veränderungen der Einstellungs- und Verhaltensmuster in der Türkei und unter den Migranten in Westeuropa in den vergangenen Dekaden weitgehend ähnlich oder möglicherweise konvergierend verliefen und daher heute zu so ähnlichen Befunde führen. Dann wäre nicht Beständigkeit in der Migration, sondern „Konvergenz“ oder „Wandel im Konvoi“ die korrektere Interpretation. Obwohl das vorliegende Studiendesign die Herkunftseffekte weitgehend minimieren kann, bleiben schließlich Selektionseffekte eine Alternativerklärung für die (wenigen) beobachteten Differenzen zwischen Migranten und Familien im Herkunftsland. Dies betrifft v.a. die Selektion bei der Entscheidung für die Migration nach Europa, selbst wenn diese inzwischen schon einige Jahrzehnte zurückliegt.

Trotz dieser Einschränkungen stellt die LineUp-Studie eine wichtige Weiterentwicklung für die empirische Migrations- und Familienforschung dar. Mit der „Herkunftslandperspektive“" wird eine bisher viel zu wenig berücksichtigte Forschungslücke adressiert und ein neues Forschungsfeld betreten. 


\section{Danksagung}

Die LineUp-Studie wird finanziert durch das NORFACE Research Programme on Migration in Europe - Social, Economic, Cultural and Policy Dynamics.

\section{Literatur}

Albertini, M., Kohli, M. \&Vogel, C. (2007). Intergenerational transfers of time and money in European families: Common patterns - different regimes? Journal of European Social Policy, 17, S. 319-334.

Arends-Toth, J. \& Van de Vijver, F. J. R. (2008). Family relationships among immigrants and majority members in the Netherlands: The role of acculturation. Applied Psychology: An International Review 57,3 , S. 466-487.

Ataca, B., Kağıtçıbaşı, C. \& Diri, A. (2005). The Turkish family and the value of children: Trends over time. In: Trommsdorff, G./Nauck, B. (Hrsg.), The value of children in cross-cultural perspective. Case studies from eight societies. Lengerich: Pabst Science: 91-119.

Attias-Donfut, C./Wolff, F.-C. (2008). Patterns of intergenerational transfers among immigrants in France: A comparative perspective. In: Saraceno, C. (Hrsg.), Families, ageing and social policy. Intergenerational solidarity in European welfare states. Cheltenham, Northhampton: Edward Elgar, S. 259-284.

Aytaç, I. (1998). Intergenerational living arrangements in Turkey. Journal of Cross-Cultural Gerontology, S. 241-264.

Baykara-Krumme, H. (2012). Die Bedeutung der Migrationserfahrung für die soziale Einbindung im Alter - Konzeptionelle Überlegungen und empirische Befunde. In: Baykara-Krumme, H., MotelKlingebiel, M. \& Schimany, P. (Hrsg.), Viele Welten des Alterns. Ältere Migranten im alternden Deutschland. Wiesbaden: VS Verlag für Sozialwissenschaften, S. 255-287.

Baykara-Krumme, H. \& Nauck, B. (2011). Familienmigration und neue Migrationsformen: die Mehrgenerationenstudie „LineUp“. In: Eryılmaz, A. \& Lissner, C. (Hrsg.): Geteilte Heimat - Paylaşılan Yurt. 50 Jahre Migration aus der Türkei. Essen: Klartext Verlag, S. 136-146.

Baykara-Krumme, H., Klaus, D. \& Steinbach, A. (2011). Generationenbeziehungen in Deutschland. Ein Vergleich der Beziehungsqualität in einheimischen deutschen Familien, Familien mit türkischem Migrationshintergrund und Aussiedlerfamilien. In: Brüderl, J., Castiglioni, L. \& Schumann, N. (Hrsg.), Partnerschaft, Fertilität und intergenerationale Beziehungen. Würzburg: Ergon, S. 259-286.

Baykara-Krumme, H. (2008). Immigrant families in Germany. Intergenerational solidarity in later life. Berlin: Weissensee Verlag.

Bengtson, V. L. (1975). Generation and family effects in value socialization. American Sociological Review, 40, 3, S 358-371.

Bengtson, V. \& Roberts, R. E. L. (1991). Intergenerational solidarity in aging families: An example of formal theory construction. Journal of Marriage and the Family, 53, S. 856-870.

Bengtson, V. L. \& Allen, K. R. (1993). The life course perspective applied to families over time. In: Boss, P., Doherty, W. J., Larossa, R., Schumm, W. R. \& Steinmetz, S. K. (Hrsg.), Sourcebook of family theories and methods. New York: Springer US, S. 469-504.

Blome, A., Keck, W. \& Alber, J. (2008). Generationenbeziehungen im Wohlfahrtsstaat. Wiesbaden: VS Verlag für Sozialwissenschaften.

Burr, J. A. \& Mutchler, J. E. (1999). Race and ethnic variation in norms of filial responsibility among older persons. Journal of Marriage and the Family, 61, S. 647-687.

Cong, Z. \& Silverstein, M. (2011). Intergenerational exchange between parents and migrant and nonmigrant sons in rural China. Journal of Marriage and Family, 73, S. 93-104.

Dietzel-Papakyriakou, M. (1993). Altern in der Migration. Die Arbeitsmigranten vor dem Dilemma: zurückkehren oder bleiben? Stuttgart: Ferdinand Enke Verlag.

De Valk, H./Schans, D. (2008). 'They ought to do this for their parents': Perceptions of filial obligations among immigrant and Dutch older people. Ageing \& Society, 28, S. 49-66. 
Duben, A. (1982). The significance of family and kinship in urban Turkey. In: Kağıtçıbaşı, Ç. (Hrsg.), Sex roles, family and community in Turkey. Indiana: Indiana University, S. 73-100.

Durkheim, E. (1921). La famille conjugale. Revue philosophique 91-92, 1-24. In: Simpson, G. (1965), A Durkheim fragment. American Journal of Sociology, 70, S. 527-536.

El-Menouar, Y. \& Fritz, M. (2009). Sozioökonomische Entwicklung und Wertvorstellungen in elf Regionen der Türkei. Kölner Zeitschrift für Soziologie und Sozialpsychologie, 61, S. 535-561.

Van Gaalen, R. I. \& Dykstra, P.A: (2006). Solidarity and conflict between adult children and parents: A latent class analysis. Journal of Marriage and Family, 68, 4, S. 947-960.

Gans, D. \& Silverstein, M. (2006). Norms of filial responsibility for aging parents across time and generations. Journal of Marriage and Family, 68, 4, S. 961-976.

Goode, W. J. (1970). World revolution and family patterns. New York: The Free Press.

Herwartz-Emden, L. (2000). Einleitung: Geschlechterverhältnis, Familie und Migration. In: HerwartzEmden, L. (Hrsg.). Einwandererfamilien. Osnabrück: Universitätsverlag Rasch, S. 9-52.

Hofstede, G. (2001). Culture's consequences: Comparing values, behaviours, institutions and organizations across nations. Thousand Oaks, CA: Sage.

Hubert, S.. Althammer, J. \& Korucu-Rieger, C. (2009). Sozialdemographische Merkmale und psychophyisches Befinden älterer türkischer Migrantinnen und Migranten in Deutschland. Eine Untersuchung auf Basis der Haupt- und Zusatzbefragung des Generations and Gender Survey der ersten Welle. Berlin: Pro Business.

Kağıtçıbaşı, C. (1996). Family and human development across cultures: A view from the other side. Mahwah, NJ: Lawrence Erlbaum Associates.

Kalaycıoğlu, S. \& Rittersberger-Tılıç, H. (2000). Intergenerational solidarity networks of instrumental and cultural transfers within migrant families in Turkey. Ageing \& Society, 20, 05, S. 523-542.

Kalmijn, M. (2006). Educational inequality and family relationships: Influences on contact and proximity. European Sociological Review, 22, 1, S. 1-16.

Künemund, H. \& Vogel, C. (2006). Öffentliche und private Transfers und Unterstützungsleistungen im Alter - "crowding out" oder "crowding in"? Zeitschrift für Familienforschung/Journal of Family Research, 18, S. 269-289.

Matthai, I. (2005). Die „,vergessenen“ Frauen aus der Zuwanderergeneration: Zur Lebenssituation alleinstehender Migrantinnen im Alter. Wiesbaden: VS Verlag für Sozialwissenschaften.

Merz, E.-M., Oort, F.J., Özeke-Koçabaş, E. \& Schüngel, C. (2009): Intergenerational family solidarity: Value differences between immigrant groups and generations. Journal of Family Psychology, 23, 3, S. 291-300.

Nauck, B. (1988). Inter- und intragenerativer Wandel in Migrantenfamilien. Soziale Welt, 4, S. 504-521.

Nauck, B. (1997). Migration and intergenerational relations: Turkish families at home and abroad. In: Wlesowod, W. I. (Hrsg.), Multiculturalism in North America and Europe: Comparative perspectives on interethnic relations and social incorporation. Toronto: Canadian Scholars' Press, S. 435465.

Nauck, B. (2002). Dreißig Jahre Migrantenfamilien in der Bundesrepublik. Familiärer Wandel zwischen Situationsanpassung, Akkulturation, Segregation und Remigration. In: Nave-Herz, R. (Hrsg.), Kontinuität und Wandel der Familie in Deutschland. Eine zeitgeschichtliche Analyse. Stuttgart: Lucius \& Lucius, S. 315-339.

Nauck, B. \& Suckow, J. (2006). Intergenerational relationships in cross-cultural comparison: How social networks frame intergenerational relations between mothers and grandmothers in Japan, Korea, China, Indonesia, Israel, Germany, and Turkey. Journal of Family Issues, 27, S. 1159-1185.

Nauck, B. \& Klaus, D. (2005). Families in Turkey. In: Adams, B. N. \& Trost, J. (Hrsg.), Handbook of world families. Thousand Oaks, London: Sage, S. 364-388.

Park, R.E. (1964). Race and culture. New York: The Free Press.

Parsons, T. \& Bales, R.F. (1956). Family. Socialization and interaction process. London: Routledge \& Kegan Paul.

Phalet, K. \& Schönpflug, U. (2001). Intergenerational transmission of collectivism and achievement values in two acculturation contexts: The case of Turkish families in Germany and Turkish and Moroccan families in the Netherlands. Journal of Cross-Cultural Psychology, 32, 2, S. 186-201. 
Portes, A. \& Rumbaut, R. (2001). Legacies. The story of the immigrant second generation. Berkely: University of California Press.

Portes, A. (2003). Conclusion: Theoretical convergencies and empirical evidence in the study of immigrant transnationalism. International Migration Review, 37, 3, S. 874-892.

Reher, D. S. (1998): Family ties in Western Europe: Persistent contrasts. Population and Development Review, 24, 2, S. 203-234.

Schans, D. \& Komter, A. (2010). Ethnic differences in intergenerational solidarity in the Netherlands. Journal of Aging Studies, 24, S.194-203.

Schrader, A., Nikles, B.W. \& Griese, H. M. (1979). Die zweite Generation. Sozialisation und Akkulturation ausländischer Kinder in der Bundesrepublik. Königstein: Athenäum Verlag.

Şenyürekli, A. R. \& Detzner, D. F. (2008). Intergenerational relationships in a transnational context: The case of Turkish families. Family Relations, 57, S. 457-467.

Silverstein, M. \& Attias-Donfut, C. (2010). Intergenerational relationships of international migrants in developed nations: The United States and France. In: Dannefer, D. \& Phillipson, C. (Hrsg.), The SAGE handbook of social gerontology. Los Angeles u. a.: Sage Publications, S. 177-189.

Silverstein, M. \& Chen, X. (1999). The impact of acculturation in Mexican American families on the quality of adult grandchild-grandparent relationships. Journal of Marriage and the Family, 61,1 , S. 188-198.

Steinbach, A. \& Kopp, J. (2008). Intergenerationale Beziehungen. Theoretische Diskussionen, empirische Befunde und offene Fragen. In: Huinink, J. \& Feldhaus, M. (Hrsg.), Neuere Entwicklungen in der Beziehungs- und Familienforschung - Vorstudien zum Beziehungs- und Familienentwicklungspanel (PAIRFAM). Würzburg: Ergon, S. 401-428.

Thornton, A. (2005). Reading history sideways. The fallacy and enduring impact of the developmental paradigm on family life. Chicago: University of Chicago Press.

Treas, J. \& Marcum, C. S. (2011). Diversity and family relations in an aging society. In: Settersen, R. A. \& Angel, J. L. (Hrsg.), Handbook of sociology of aging. New York u.a.: Springer, S. 131-141.

Tucci, I. \& Yıldız, S. (2012). Das Alterseinkommen von Migrantinnen und Migranten: zur Erklärungskraft von Bildungs- und Erwerbsbiografien. In: Baykara-Krumme, H., Motel-Klingebiel, A. \& Schimany, P. (Hrsg.), Viele Welten des Alterns? Ältere Migranten im alternden Deutschland. Wiesbaden, VS Verlag für Sozialwissenschaften, S. 101-126.

Zimmermann, H.-P. (2012). Altersbilder von türkischen Migrantinnen und Migranten in Deutschland im Vergleich. Islamische Grundsätze - alltägliche Sichtweise. In: Baykara-Krumme, H., MotelKlingebiel, M. \& Schimany, P. (Hrsg.), Viele Welten des Alterns. Ältere Migranten im alternden Deutschland. Wiesbaden: VS Verlag für Sozialwissenschaften, S. 315-337.

Zhou, M. (1997). Growing up American: The challenge confronting immigrant children and children of immigrants. Annual Review of Sociology, 23, S. 63-95.

Eingereicht am/Submitted on 17.07.2012

Angenommen am/Accepted on: 31.10.2012

Anschrift der Autorin/Address of the author:

Dr. Helen Baykara-Krumme

Technische Universität Chemnitz

Institut für Soziologe

Thüringer Weg 9

09126 Chemnitz

Deutschland/Germany

E-Mail: helen.baykara@soziologie.tu-chemnitz.de 